\title{
Arthroscopic Burring of Exposed Cement Following Curettage and Cavity Filling Cementation for Chondroblastoma of the Proximal Tibia
}

\author{
Jong-Hoon Park, $\mathrm{MD}^{1}$, In-Jung Chae, $\mathrm{MD}^{1}$, Seung-Beom Han, $\mathrm{MD}^{1}$, and Dae-Hee Lee, $\mathrm{MD}^{2}$ \\ ${ }^{1}$ Department of Orthopaedic Surgery, Korea University Anam Hospital, Korea University College of Medicine, Seoul; ${ }^{2}$ Department of Orthopaedic Surgery, Samsung \\ Medical Center, Sungkyunkwan University School of Medicine, Seoul, Korea
}

\begin{abstract}
Chondroblastoma of the proximal tibia is difficult to treat because of its epiphyseal predilection. This condition can be treated by curettage, which results in immediate restoration of stability and a reduced recurrence rate, followed by cement filling of the bone defect. Nevertheless, contact with cement can damage articular cartilage, potentially leading to severe knee osteoarthritis. Most previous reports regarding this complication described patients with giant cell tumors of the proximal tibia. We present here a patient who underwent arthroscopic treatment for cement exposure caused by articular cartilage loss of the tibial plateau, which occurred after initial curettage and cementation for chondroblastoma of the proximal tibia. To our knowledge, this is the first report on arthroscopic treatment of this condition.
\end{abstract}

Keywords: Tibia, Chondroblastoma, Arthroscopy, Polymethyl methacrylate

Chondroblastoma is a rare benign bone tumor with the potential for aggressive local growth that tends to occur in the epiphyses of long tubular bones such as the distal femur and proximal tibia $^{1)}$. Treatment of chondroblastoma of the proximal tibia involving subchondral bone is challenging due to the difficulty of achieving adequate curettage of the tumor while preserving the articular cartilage, especially in cases of extensive subchondral bone involvement. Curettage and cementation have been used to treat patients with this type of periarticular tumor, including giant cell tumors and chondroblastomas ${ }^{2,3)}$. Although the index surgery has several advantages, including immediate restoration

Received June 15, 2014; Revised December 4, 2014;

Accepted January 20, 2015

Correspondence to: Dae-Hee Lee, MD

Department of Orthopaedic Surgery, Samsung Medical Center, Sungkyunkwan University School of Medicine, 81 Ilwon-ro, Gangnamgu, Seoul 135-710, Korea

Tel: +82-2-3410-3507, Fax: +82-2-3410-0061

E-mail: eoak22@empal.com

This is an Open Access article distributed under the terms of the Creative Commons Attribution Non-Commercial License (http://creativecommons.org/licenses/by-nc/3.0/) which permits unrestricted non-commercial use, distribution, and reproduction in any medium, provided the original work is properly cited. of stability, avoidance of internal fixation, and decreased local recurrence rates, knee osteoarthritis may occur due to the exposure of articular cartilage to cement, eventually resulting in loss of articular cartilage ${ }^{2-4)}$. This complication is of particular concern in patients with tumors of the proximal tibia, especially in patients with giant cell tumors ${ }^{4,5}$. We describe here a patient who experienced full-thickness cartilage loss due to cement exposure after initial curettage and cementation for chondroblastoma; this patient was successfully treated by arthroscopic burring of the exposed cement.

\section{Case Report}

A 20-year-old male was referred to our clinic due to a gradual increase in pain along with swelling in his right knee. These symptoms had first appeared 1 year earlier and had worsened during the previous 3 months. Five years prior to presentation, he underwent a curettage and cavity filling cementation with polymethyl methacrylate (PMMA) cement for a chondroblastoma of the proximal tibia at another hospital. His postoperative course was uneventful and he continued to work at his job and performed light exercises comfortably. Approximately 5 years after surgery, the patient reported insidiously progressive knee pain 
with restricted range of motion, as well as intermittent clicking at various knee positions. He had no history of major knee trauma.

Physical examination revealed moderate swelling and pain around the knee joint without heating. The range of knee motion was restricted to an extension of $-15^{\circ}$ and a flexion of $110^{\circ}$. Wasting of the quadriceps muscles was observed. Plain radiographs showed no evidence of local tumor relapse, but suggested intraarticular extension of PMMA. Computed tomography (CT) of the knee showed protrusion of cement into the knee joint through a broken subchondral bone and articular cartilage in both the medial and lateral tibial spines of the tibia and scraping of the lateral aspect of the intercondylar notch (Fig. 1). Arthroscopy revealed a full-thickness loss of articular cartilage in the area of the lateral tibial spine, the absence of subchondral bone, and a sharp bump of the exposed PMMA cement from the previous surgery, with bony erosion of the lateral wall of the intercondylar notch (Fig. 2A).

The patient was treated by debridement of the articular cartilage remaining on the area of the lateral tibial spine, followed by shaving off and contouring of the PMMA cement bump with an arthroscopic burr to prevent scratching of the lateral surface of the intercondylar notch (Fig. 2B). The cement that protruded above the articular cartilage of the tibial plateau was removed carefully, so as not to destroy the cement bump that filled in the subchondral cavity below the surface of the articular cartilage of the tibial plateau. Remnant cement debris created by burring was avoided by using the outer sheath of the shaver from which the blade had been removed. The cement debris was swept away in
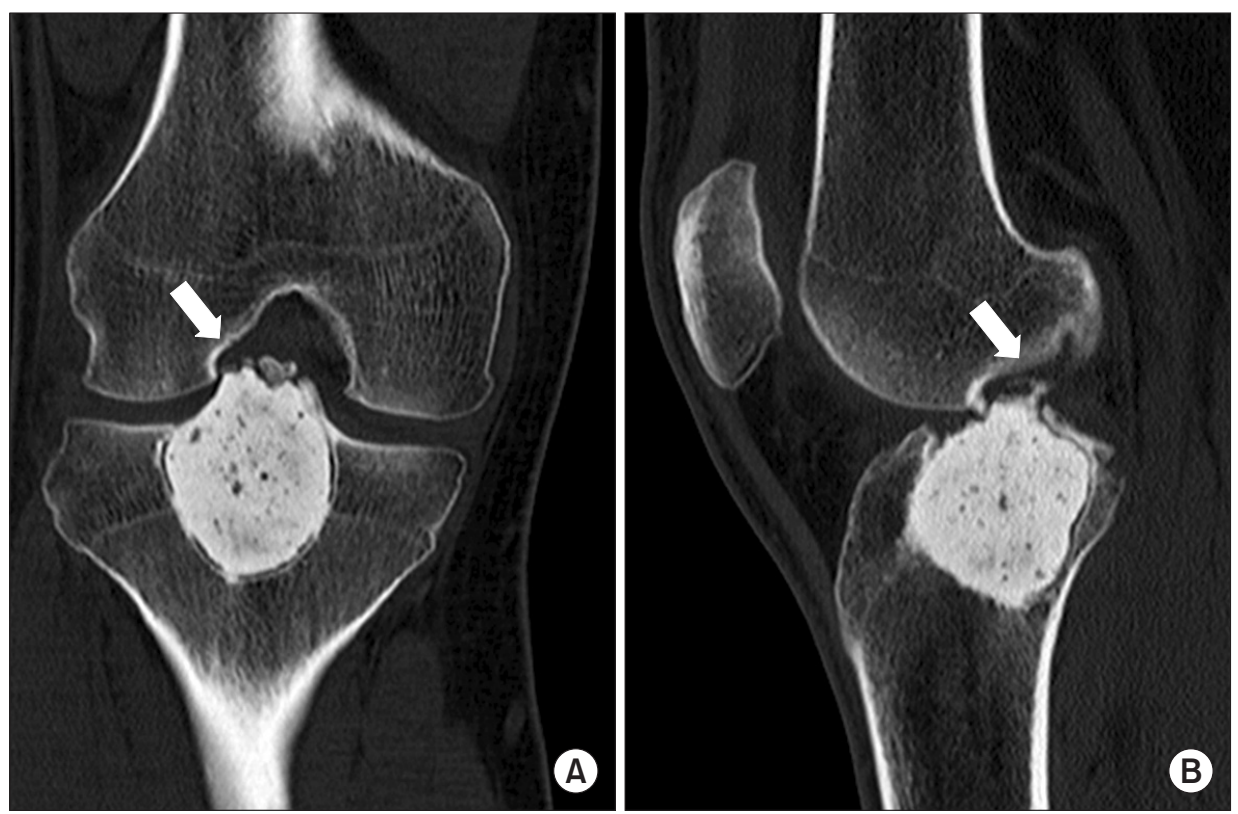

B
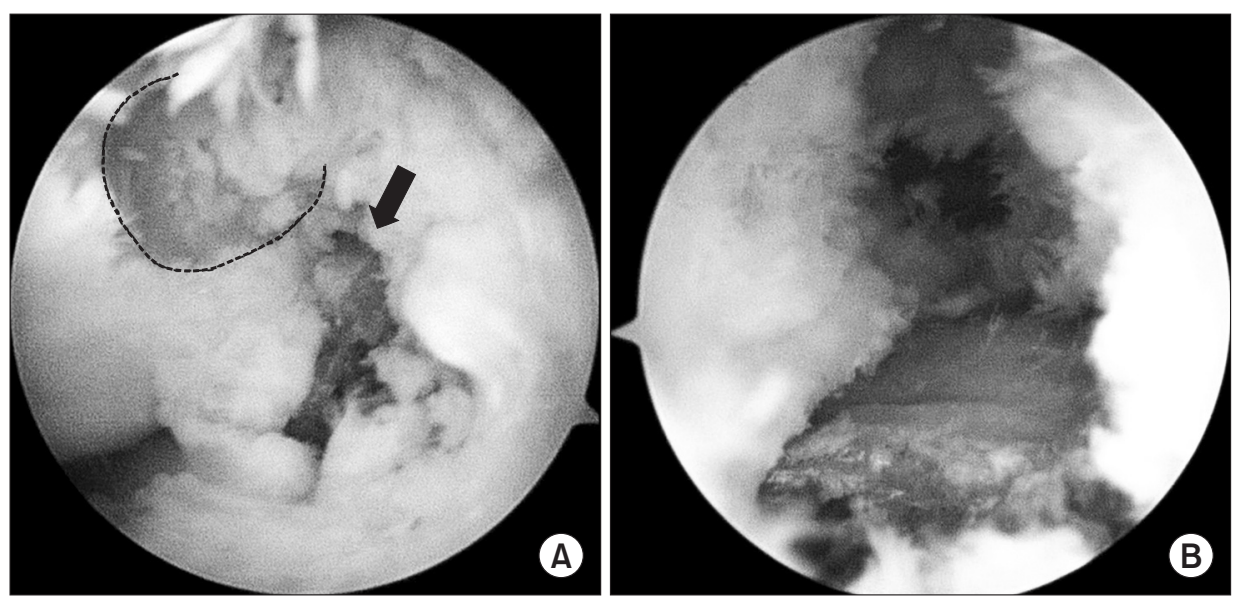

Fig. 2. (A) Arthroscopic view showing cement extrusion (arrow) through the articular cartilage of the tibial plateau and a bony defect in the lateral aspect of the intercondylar notch (dotted line) resulting from scraping by extruded cement. (B) Arthroscopic view after chondroplasty and removal of the exposed cement. 

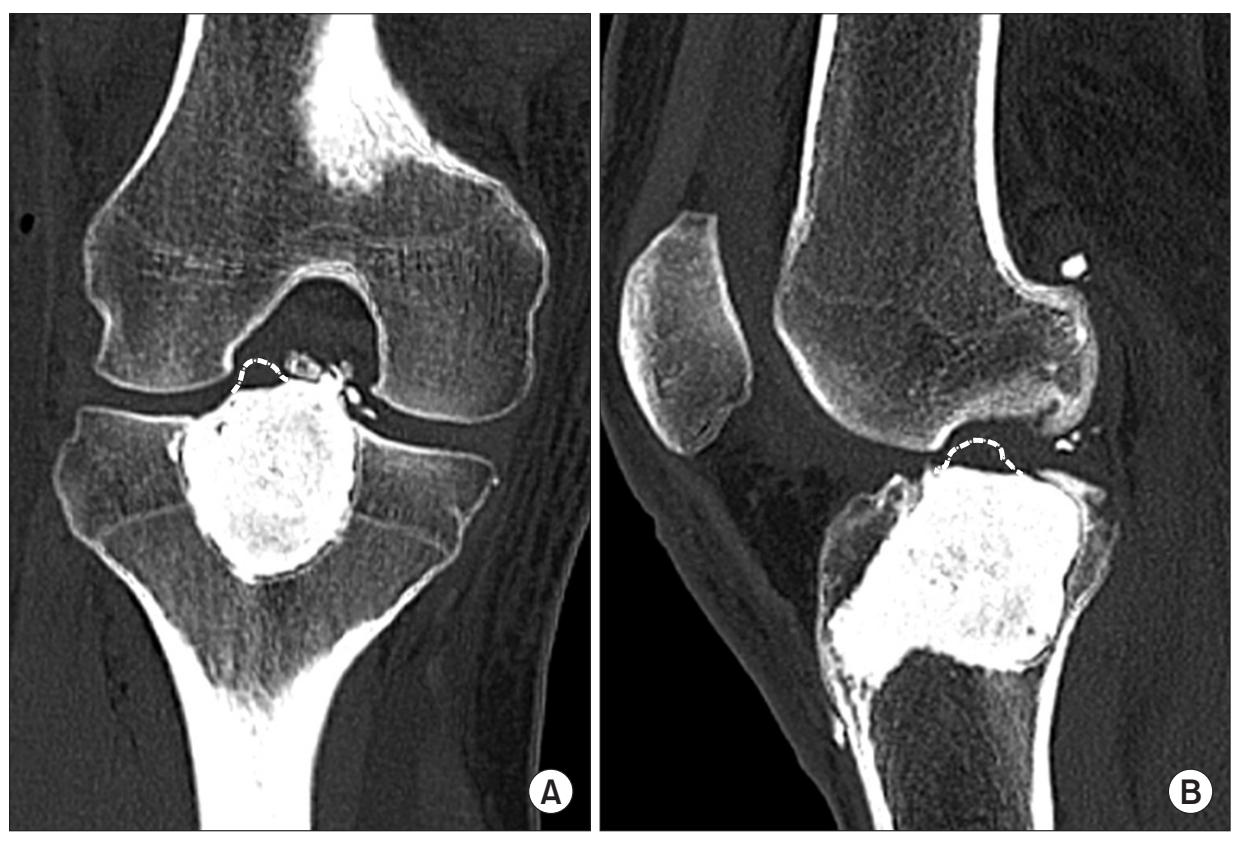

Fig. 3. Postoperative coronal (A) and sagittal (B) computed tomographic images showing the removal of the sharp cement bump (dotted lines) that impinged the lateral aspect of the intercondylar notch.

the irrigation fluid flowing from the knee joint through the hole of the outer sheath of the shaver. Postoperative CT showed that the cement bump had been removed (Fig. 3). Postoperatively, the patient was allowed to bear weight as tolerated with crutches and without limiting knee motion. At first follow-up on postoperative day 7 , the patient showed full knee motion without pain. At 26 months, the patient showed maintenance of full knee motion and was pain-free.

\section{Discussion}

Chondroblastoma is a rare benign bone tumor, accounting for approximately $1 \%$ of all primary bone tumors ${ }^{6)}$. In contrast to giant cell tumors, chondroblastomas are frequent in children and adolescents and are usually located in the epiphysis of a long bone, such as the humerus, distal femur, and proximal tibia ${ }^{1)}$. Chondroblastomas in immediate proximity to joints are difficult to treat due to local physeal involvement and have a relatively high recurrence rate of $10 \%-35 \%{ }^{7)}$. There is no general consensus for standard treatment of chondroblastoma. Curettage is the usual surgical treatment option; it may be used alone, in conjunction with bone grafting or packing of the cavity with poly (methyl acrylate), or coupled with cryosurgery ${ }^{8)}$.

Simple curettage of chondroblastomas ${ }^{7)}$ and giant cell tumors ${ }^{9)}$, however, has been associated with a relatively high recurrence rate. The recurrence rate can be lowered by filling the bone defect with cement after curettage; this lowering of the recurrence rate is due to the toxic properties of the methylmethacrylate mono- mer and the necrotic effect of heat generated during polymerization on tumor cells ${ }^{2}$. Cementation also preserves the dynamic stability of the knee, allowing rapid loading, and enables early radiologic detection of recurrence as lysis at the cement-bone interface ${ }^{3,4)}$. Despite its ease of use and low cost, however, contact of the cement with the cartilage can damage the latter, possibly leading to osteoarthritis.

To date, only two case reports have described arthroscopic treatment of cement exposure after curettage and cementation for a giant cell tumor of the proximal tibia. Two patients with symptomatic full-thickness tibial articular loss after curettage and PMMA reconstruction of a giant cell tumor of the proximal tibia underwent arthroscopic chondroplasty and planing of the exposed cement ${ }^{5)}$. Another patient experienced severe osteoarthritis with varus deformity due to collapse of the medial wall of the proximal tibia approximately 4 years after curettage, cryosurgery, and cementation for a giant cell tumor of the proximal tibia ${ }^{4)}$. In this patient, the knee joint destruction and bone defect were so severe as to require knee joint reconstruction, including removal of the cement, correction of alignment by tibial osteotomy, reconstruction of the subchondral bone, and distraction osteogenesis using the Ilizarov apparatus. In this patient, arthroscopy before knee joint reconstruction showed the protrusion of cement into the intra-articular knee joint. Similarly, arthroscopy in our patient showed the absence of articular cartilage and of subchondral bone, primarily in the area of the lateral tibial spine, and a sharp bump of the exposed PMMA cement. This resulted from initial oncologic management, which included shaving off the lateral 
aspect of the intercondylar notch of the distal femur as a kissing lesion. We, therefore, arthroscopically flattened the protruding cement using a burr after chondroplasty. We also took care to completely remove the cement debris created during burring, because large sized cement particles could directly scratch the articular cartilage, while small sized cement particles could indirectly induce osteolysis, similar to the third body wear reaction. Cement debris was removed by drainage of the irrigation fluid through the hole of the outer sheath of the shaver.

Although we could not accurately determine the cause of cement protrusion from the proximal tibial cortex, it may have been due to thermal damage by the cement to the subchondral bone and cortex. Articular cartilage is likely to be at risk of thermal damage if the distance between it and the cement is too close due to the underlying subchondral layer being too thin $(<2$ $\mathrm{mm})^{10)}$. If this situation cannot be avoided, countermeasures are available. First, cementing can be performed in stages. A small amount of cement with limited heat generation capacity could be spread onto the subchondral cavity wall, followed later by a larger batch of cement to fill the remaining cavity. Second, cooling of surrounding bone and soft tissue before and during cement implantation may reduce thermal damage to the articular cartilage. Finally, an autogenous bone graft or allograft could be used to reconstruct subchondral bone between the articular cartilage and the cement. These bone grafts may reduce subchondral bone collapse by a shock absorption effect and act as a barrier to prevent thermal damage by cement polymerization to the articular cartilage.

In conclusion, cement filling of a bone defect after curettage of a chondroblastoma of the proximal tibia should be performed with caution. In these patients, the articular cartilage may be thin due to unavoidable extensive subchondral bone loss, which can be exacerbated by contact with the cement. Arthroscopic burring may be effective in removing cement extruded into the knee joint, but long-term follow-up is required to evaluate whether such patients develop osteoarthritis of the knee.

\section{Conflict of Interest}

No potential conflict of interest relevant to this article was reported.

\section{References}

1. Robbin MR, Murphey MD. Benign chondroid neoplasms of bone. Semin Musculoskelet Radiol. 2000;4:45-58.

2. Fraquet N, Faizon G, Rosset P, Phillipeau J-, Waast D, Gouin F. Long bones giant cells tumors: treatment by curretage and cavity filling cementation. Orthop Traumatol Surg Res. 2009; 95:402-6.

3. Lehner B, Witte D, Weiss S. Clinical and radiological longterm results after operative treatment of chondroblastoma. Arch Orthop Trauma Surg. 2011;131:45-52.

4. Wakitani S, Imoto K, Saito M, Yamamoto T, Kawabata H. A case report: reconstruction of a damaged knee following treatment of giant cell tumor of the proximal tibia with cryosurgery and cementation. Osteoarthritis Cartilage. 2002;10:402-7.

5. Tejwani SG, Hame SL, Eckardt JJ. Subchondral giant-cell tumor of the proximal tibia: arthroscopic treatment for accelerated articular cartilage and meniscal degeneration in two patients. Arthroscopy. 2004;20:644-9.

6. Springfield DS, Capanna R, Gherlinzoni F, Picci P, Campanacci M. Chondroblastoma: a review of seventy cases. J Bone Joint Surg Am. 1985;67:748-55.

7. Ramappa AJ, Lee FY, Tang P, Carlson JR, Gebhardt MC, Mankin HJ. Chondroblastoma of bone. J Bone Joint Surg Am. 2000;82:1140-5.

8. Suneja R, Grimer RJ, Belthur M, Jeys L, Carter SR, Tillman RM, Davies AM. Chondroblastoma of bone: long-term results and functional outcome after intralesional curettage. J Bone Joint Surg Br. 2005;87:974-8.

9. Campanacci M, Baldini N, Boriani S, Sudanese A. Giant-cell tumor of bone. J Bone Joint Surg Am. 1987;69:106-14.

10. Radev BR, Kase JA, Askew MJ, Weiner SD. Potential for thermal damage to articular cartilage by PMMA reconstruction of a bone cavity following tumor excision: A finite element study. J Biomech. 2009;42:1120-6. 